\title{
Factors affecting choice of sponsoring institution for residency among medical students in Singapore
}

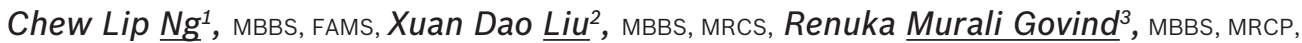 \\ Jonathan Wei Jian Tan ${ }^{4}$, MBBS, Shirley Beng Suat Ooij, ${ }^{5,6}$, MBBS, FRCSEd, Sophia Archuleta7,8, MD
}

INTRODUCTION Postgraduate medical education in Singapore underwent a major transition recently, from a British-style system and accreditation to a competency-based residency programme modelled after the American system. We aimed to identify the relative importance of factors influencing the choice of sponsoring institution (SI) for residency among medical students during this transition period.

METHODS A questionnaire-based cross-sectional study of Singapore undergraduate medical students across all years of study was performed in 2011. Participants rated the degree of importance of 45 factors (including research, academia and education, marketing, reputation of faculty, working conditions, posting experience and influence by peers/seniors) to their choice of SIs on a five-point Likert scale. Differences in gender and seniority were compared.

RESULTS 705 out of 1,274 students completed the survey (response rate $55.3 \%$ ). The top five influencing factors were guidance by mentor $(4.48 \pm 0.74)$, reputation for good teaching $(4.46 \pm 0.76)$, personal overall experience in $\mathrm{SI}$ (4.41 \pm 0.88$)$, quality of mentorship and supervision $(4.41 \pm 0.75)$, and quality and quantity of teaching $(4.37 \pm 0.78)$. The five lowest-rated factors were social networking ( $2.91 \pm 1.00)$, SI security ( $3.01 \pm 1.07)$, open house impact (3.15 \pm 0.96$)$, advertising paraphernalia $(3.17 \pm 0.95)$ and research publications $(3.21 \pm 1.00)$. Female students attributed more importance to security and a positive working environment. Preclinical students rated research and marketing aspects more highly, while clinical students valued a positive working environment more.

CONCLUSION Quality of education, mentorship, experiences during clerkship and a positive working environment were the most important factors influencing the choice of SI.

Keywords: ACGME-I, factors, medical students, residency

\section{INTRODUCTION}

Between 2009 and 2012, postgraduate medical education in Singapore underwent major reform, wherein a transition was made from the existing British-style Graduate Medical Education system to a competency-based residency programme modelled after the American system, which was accredited by ACGME-I (Accreditation Council for Graduate Medical EducationInternational).(1)

Important consequences of this change were that: (a) residents no longer rotated through various hospitals across Singapore under a national training programme; and (b) hospitals no longer functioned as independent postgraduate training institutes. Coalitions of hospitals - termed sponsoring institutions (SIs) and participating institutions (PIs) - became clusters that were responsible for residents under their charge, and residents experienced rotations only within each cluster of SIs and PIs throughout the duration of residency. Therefore, the matching of residents to SIs now has significant implications for both the applicant and SI. With this major transition, it has become crucial for both parties to understand the factors that influence the choice of residency programmes among medical students: the SIs would want to improve their pedagogy and training facilities to attract the best possible talent, while residency applicants want to know what each SI offers and which one is best suited to their training needs and aspirations.

The primary aim of this study was to identify and establish the relative importance of factors influencing the choice of SIs among medical students for residency. A secondary aim was to identify variations in these influencing factors based on gender and seniority in medical school. In particular, it was deemed critical to understand the prevailing attitudes of senior medical students, as these were candidates who could enter residency directly upon graduation from medical school.

\section{METHODS}

We performed a questionnaire-based cross-sectional study of students across all five years of medical school who were enrolled at the Yong Loo Lin School of Medicine, National University of Singapore, during the academic year of $2011 / 2012$. The total student population was 1,274 . Students were recruited by study administrators in their respective lecture halls during curriculum hours. Verbal and written instructions were given, and all students were informed verbally and in writing that participation was anonymous and voluntary. They were reassured that participation in the survey would not affect their future application for residency. All questionnaires were handed out and completed in hard copy.

\footnotetext{
${ }^{1}$ Department of Ear, Nose and Throat - Head and Neck Surgery, Ng Teng Fong General Hospital, ${ }^{2}$ Department of Otolaryngology-Head and Neck Surgery, National University Health System, ${ }^{3}$ Internal Medicine, National Healthcare Group, ${ }^{4}$ Ministry of Health Holdings, ${ }^{5}$ Emergency Medicine Department, National University Hospital, ${ }^{6}$ National University Health System Residency Programme, ${ }^{7}$ Department of Medicine, NUS Yong Loo Lin School of Medicine, National University of Singapore, ${ }^{8}$ Division of Infectious Diseases, National University Health System, Singapore

Correspondence: Dr Ng Chew Lip, Associate Consultant, Department of Ear, Nose and Throat - Head and Neck Surgery, Ng Teng Fong General Hospital, 1 Jurong East Street 21 , Singapore 609606.ngchewlip@gmail.com
} 
Basic demographic information was obtained, including year of study and gender. No personal identifiers were collected from the participants. Overall, 45 factors divided into 11 categories were studied. The categories included: physical characteristics of the institutions, research, academia and education, marketing, residency application process, reputation of chief administrators (programme directors and designated institution officials), working conditions, participation in feedback, welfare, experience as medical students and influence by peers/seniors. For each factor, students were asked to indicate its degree of importance to their choice of SI on a five-point Likert scale, with 1 being 'least important' and 5 being 'very important'. The list of factors was derived with reference to previous studies, ${ }^{(2-4)}$ as well as from pre-study focus group discussions with programme directors, residents and residency administrators. The Cronbach's alpha reliability coefficients of ten of the 11 categories ranged from $0.676-0.920$, with the exception of 'physical factors of $\mathrm{SI}^{\prime}$ (Cronbach's alpha 0.363), likely because of the disparate nature of its three factors (proximity, security and site of hospital). Overall, the questionnaire showed good internal consistency.

We analysed the data using IBM SPSS Statistics version 20.0 (IBM Corp, Armonk, NY, USA). The Likert scale ratings (mean rating \pm standard deviation) were normally distributed, with skewness in the range of -1.786 to -0.176 and kurtosis in the range of -0.917 to 1.229 . Student's $t$-test was used to compare the differences between the mean ratings of each factor. For comparing binomial variables, chi-square test was used. Statistical significance was defined as $p<0.05$. The student population was compared by dividing it into the preclinical (Year 1-2) and clinical (Year 3-5) groups. This dichotomy was based on the distinction that clinical students were likely to have experienced hospitalbased rotations, which would have exposed them to work life in at least one SI.

Our study protocol was granted an exemption from full review by the Domain Specific Review Board (Domain F) of the National Healthcare Group (DSRB Reference 2015/00921), Singapore.

\section{RESULTS}

In total, 718 completed questionnaires from 1,274 medical students were collected. 13 respondents who did not indicate their gender were excluded from analysis, so that the overall response rate was $55.3 \%$ (Table I). Gender distribution was comparable, with 362 (51.3\%) male students and 343 (48.7\%) female students. While the participation rate was significantly higher among final-year students (95.6\%), it was diminished among fourth-year students (28.0\%). This was perhaps because our study period coincided with the elective rotations, which many students opt to do overseas.

The mean ratings for individual factors are presented in Fig. 1. The top five factors were guidance by mentor $(4.48 \pm 0.74)$, reputation for good teaching $(4.46 \pm 0.76)$, personal experience in SI (4.41 \pm 0.88$)$, quality of mentorship and supervision (4.41 $\pm 0.75)$, and quality and quantity of teaching (4.37 \pm 0.78$)$. The five lowest-rated factors, meanwhile, were social and networking opportunities $(2.91 \pm 1.00)$, SI security $(3.01 \pm 1.07)$, impact of
Table I. Demographics and response rate $(n=1,274)$.

\begin{tabular}{|c|c|c|c|c|c|}
\hline \multirow[t]{3}{*}{ Year } & \multicolumn{4}{|c|}{ No. } & \multirow{3}{*}{$\begin{array}{l}\text { Response } \\
\text { rate (\%) }\end{array}$} \\
\hline & \multicolumn{3}{|c|}{ Study sample } & \multirow[t]{2}{*}{ Class size } & \\
\hline & Male & Female & Total & & \\
\hline 1 & 57 & 73 & 130 & 242 & 53.7 \\
\hline 2 & 83 & 77 & 160 & 260 & 61.5 \\
\hline 3 & 58 & 45 & 103 & 264 & 39.0 \\
\hline 4 & 33 & 39 & 72 & 257 & 28.0 \\
\hline 5 & 131 & 109 & 240 & 251 & 95.6 \\
\hline Total & 362 & 343 & 705 & 1,274 & 55.3 \\
\hline
\end{tabular}

SI open house $(3.15 \pm 0.96)$, advertising paraphernalia from SIs $(3.17 \pm 0.95)$ and research publications $(3.21 \pm 1.00)$ (Table II).

There were variations between male and female students with respect to the ratings of a few factors. Female students attributed more importance to security (female: $3.15 \pm 1.00$; male: 2.87 $\pm 1.12 ; p<0.001)$ and a positive working environment, which included friendliness of SI (female: $4.23 \pm 0.75$; male: $4.08 \pm$ $0.89 ; p=0.015$ ), house staff morale (female: $4.30 \pm 0.71$; male: $4.13 \pm 0.85 ; p=0.004)$, working relationship among healthcare professionals (female: $4.38 \pm 0.73$; male: $4.23 \pm 0.85 ; p=0.014$ ) and being able to voice opinions (female: $3.98 \pm 0.73$; male: $3.85 \pm 0.88 ; p=0.043)$. Male students, when compared to female students, placed more value on an emphasis on training residents to become educators (female: $3.87 \pm 0.79$; male: 4.04 \pm 0.93; $\mathrm{p}=0.009$ ).

Similarly, there were multiple differences between preclinical and clinical students. Preclinical students consistently rated aspects of SIs related to research $(p<0.001)$ as being more important, in addition to marketing factors ( $p \leq 0.001)$, security $(p<0.001)$, peers' choice of SI $(p<0.001)$ and the presence of simulation facilities $(p=0.003)$. Clinical students, on the other hand, rated a positive working environment $(p<0.05)$, in particular the perceived friendliness of SI ( $p=0.016)$, house staff morale $(p=0.014)$ and working relationships $(p=0.011)$ as more important. They also placed a higher level of importance on the quality of postgraduate exam training $(p=0.011)$ and frequency of night calls $(p=0.001)$.

\section{DISCUSSION}

The transition of Singapore's postgraduate medical education from a British-style Graduate Medical Education system to an American residency system represented an unprecedented shift in the national graduate medical education landscape. Medical students graduating during this transition period are required to choose an SI or PI cluster for the entire duration of their residency. To attract the best graduates, therefore, knowledge of the prevailing attitudes of medical students on what constitutes an attractive SI has become crucial to educators involved in graduate medical education.

The top rated factors, in our study, were related to education and training quality. In particular, mentorship played a crucial role - the presence and quality of mentorship were the highest and third-highest rated factors, respectively. Mentorship is 


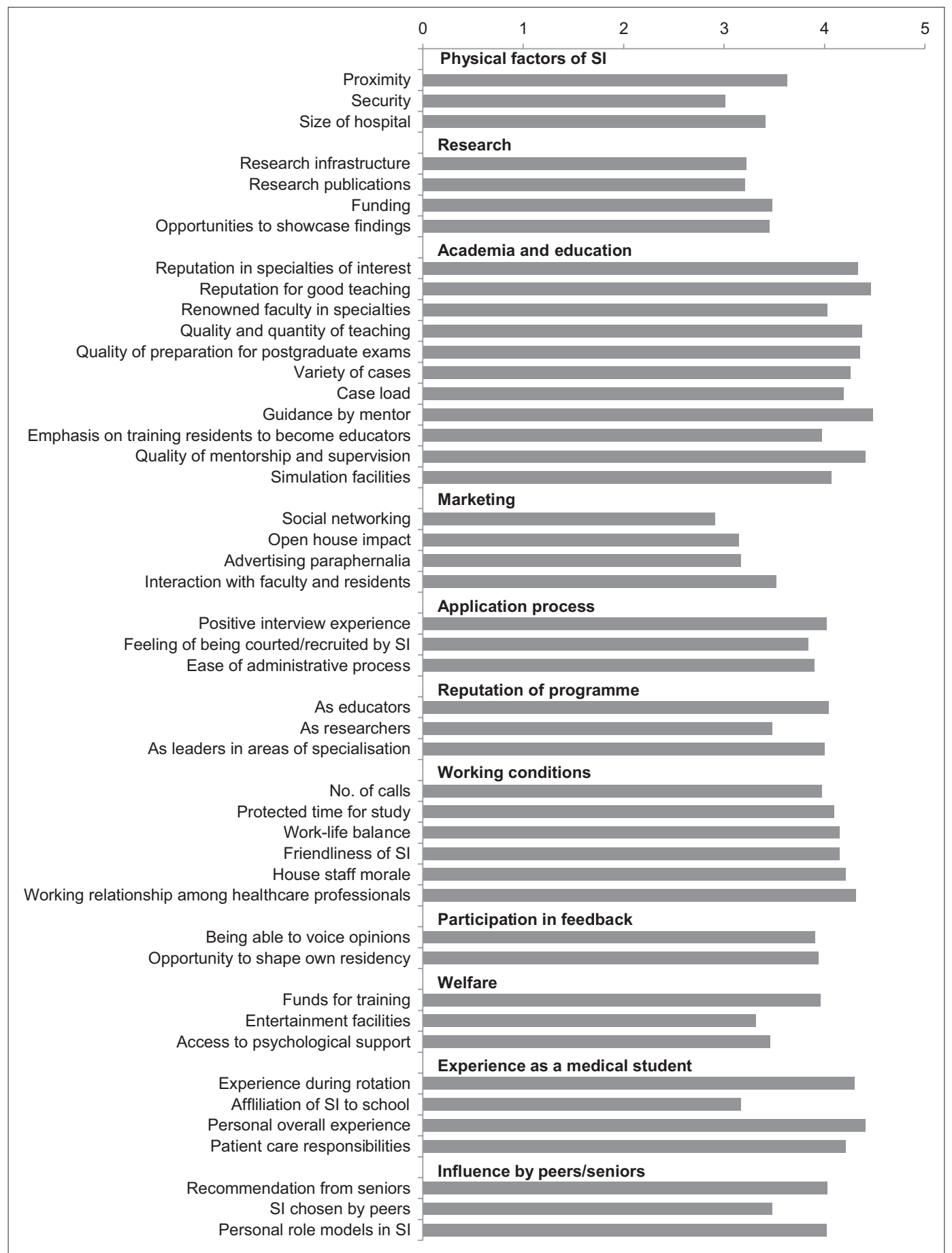

Fig. 1 Chart shows mean ratings of factors that influenced the students' choice of sponsoring institution (SI).

multifaceted; a mentor has been defined as "a faculty member who takes interest in a postgraduate trainee's career development and professional growth and does so in a non-judgemental manner". ${ }^{(5)}$ A good mentor nurtures the protégé, transfers knowledge and skills, imparts wisdom of experience and prepares for smooth succession by the next generation. A surgical mentor has the additional role of instructing on surgical technique. ${ }^{(6)}$ In a systematic review by Sambunjak et al, mentorship was reported to be an important influence on personal development, career guidance, career choice and productivity. ${ }^{(7)}$ Although mentorship in the postgraduate setting is prevalent and essential to training, it is probably lacking for medical students, with reported prevalence rates of $35 \%-54 \%$ in two studies. ${ }^{(8,9)}$ This may be perceived as a barrier by medical students or young physicians when considering a residency programme, but also presents an opportunity for SIs to build a more attractive programme. Good mentorship requires 'chemistry' between the mentor and mentee, and takes time and effort to seek out a relationship that works. ${ }^{(8)}$ Possible obstacles to mentorship of medical students may be the rapid rotations among short specialty/subspecialty postings and the uncertainty about specific specialty interests. To attract residents and stay competitive, Sls can make efforts to encourage nurturing mentormentee relationships in postgraduate medical education and develop a strong teaching culture. 
Table II. Influencing factors with significant differences according to gender and/or seniority.

\begin{tabular}{|c|c|c|c|c|c|c|c|}
\hline \multirow[t]{3}{*}{ Category/factor } & \multicolumn{7}{|c|}{ Rating (mean \pm standard deviation) } \\
\hline & \multirow[t]{2}{*}{ Overall } & \multicolumn{3}{|c|}{ Gender } & \multicolumn{3}{|c|}{ Seniority } \\
\hline & & Male & Female & p-value & $\begin{array}{l}\text { Preclinical } \\
\text { (Yr 1-2) }\end{array}$ & $\begin{array}{l}\text { Clinical } \\
\text { (Yr 3-5) }\end{array}$ & p-value* \\
\hline \multicolumn{8}{|l|}{ Physical factors of SI } \\
\hline Security & $3.01 \pm 1.07$ & $2.87 \pm 1.12$ & $3.15 \pm 1.00$ & $<0.001^{\dagger}$ & $3.21 \pm 1.05$ & $2.87 \pm 1.07$ & $<0.001^{\dagger}$ \\
\hline \multicolumn{8}{|l|}{ Research } \\
\hline Research infrastructure & $3.22 \pm 0.98$ & $3.20 \pm 1.06$ & $3.23 \pm 0.88$ & 0.641 & $3.47 \pm 0.94$ & $3.04 \pm 0.97$ & $<0.001^{\dagger}$ \\
\hline Research publications & $3.21 \pm 1.00$ & $3.15 \pm 1.09$ & $3.27 \pm 0.88$ & 0.121 & $3.41 \pm 0.95$ & $3.07 \pm 1.00$ & $<0.001^{\dagger}$ \\
\hline Funding & $3.48 \pm 1.02$ & $3.42 \pm 1.12$ & $3.54 \pm 0.89$ & 0.144 & $3.65 \pm 0.97$ & $3.36 \pm 1.03$ & $<0.001^{\dagger}$ \\
\hline \multicolumn{8}{|l|}{ Academia and education } \\
\hline $\begin{array}{l}\text { Quality of preparation for postgraduate } \\
\text { exams }\end{array}$ & $4.35 \pm 0.80$ & $4.33 \pm 0.86$ & $4.38 \pm 0.73$ & 0.343 & $4.27 \pm 0.71$ & $4.42 \pm 0.85$ & $0.011^{\dagger}$ \\
\hline $\begin{array}{l}\text { Emphasis on training residents to } \\
\text { become educators }\end{array}$ & $3.97 \pm 0.87$ & $4.04 \pm 0.93$ & $3.87 \pm 0.79$ & $0.009^{\dagger}$ & $3.92 \pm 0.82$ & $3.99 \pm 0.90$ & 0.245 \\
\hline Simulation facilities & $4.07 \pm 0.84$ & $4.10 \pm 0.87$ & $4.03 \pm 0.80$ & 0.324 & $4.18 \pm 0.76$ & $3.99 \pm 0.88$ & $0.003^{+}$ \\
\hline \multicolumn{8}{|l|}{ Marketing } \\
\hline Social networking & $2.91 \pm 1.00$ & $2.88 \pm 1.03$ & $2.94 \pm 0.97$ & 0.355 & $3.13 \pm 0.91$ & $2.76 \pm 1.03$ & $<0.001^{\dagger}$ \\
\hline Open house impact & $3.15 \pm 0.96$ & $3.09 \pm 0.98$ & $3.22 \pm 0.93$ & 0.084 & $3.29 \pm 0.85$ & $3.06 \pm 1.01$ & $0.001^{\dagger}$ \\
\hline $\begin{array}{l}\text { Advertising paraphernalia: information } \\
\text { brochures, pamphlets, emails }\end{array}$ & $3.17 \pm 0.95$ & $3.10 \pm 0.98$ & $3.24 \pm 0.91$ & 0.056 & $3.35 \pm 0.85$ & $3.04 \pm 1.00$ & $<0.001^{\dagger}$ \\
\hline \multicolumn{8}{|l|}{ Reputation of programme } \\
\hline As researchers & $3.48 \pm 0.90$ & $3.43 \pm 0.96$ & $3.54 \pm 0.83$ & 0.082 & $3.62 \pm 0.85$ & $3.39 \pm 0.92$ & $<0.001^{\dagger}$ \\
\hline \multicolumn{8}{|l|}{ Working conditions } \\
\hline No. of calls & $3.97 \pm 0.88$ & $3.94 \pm 0.89$ & $4.01 \pm 0.88$ & 0.296 & $3.85 \pm 0.80$ & $4.06 \pm 0.93$ & $0.001^{\dagger}$ \\
\hline Friendliness of SI & $4.15 \pm 0.83$ & $4.08 \pm 0.89$ & $4.23 \pm 0.75$ & $0.015^{\dagger}$ & $4.06 \pm 0.75$ & $4.21 \pm 0.88$ & $0.016^{+}$ \\
\hline House staff morale & $4.21 \pm 0.79$ & $4.13 \pm 0.85$ & $4.30 \pm 0.71$ & $0.004^{+}$ & $4.12 \pm 0.72$ & $4.27 \pm 0.83$ & $0.014^{+}$ \\
\hline $\begin{array}{l}\text { Working relationship among } \\
\text { healthcare professionals }\end{array}$ & $4.31 \pm 0.79$ & $4.23 \pm 0.85$ & $4.38 \pm 0.73$ & $0.014^{+}$ & $4.21 \pm 0.72$ & $4.37 \pm 0.84$ & $0.011^{\dagger}$ \\
\hline \multicolumn{8}{|l|}{ Participation in feedback } \\
\hline Being able to voice opinions & $3.91 \pm 0.81$ & $3.85 \pm 0.88$ & $3.98 \pm 0.73$ & $0.043^{+}$ & $3.87 \pm 0.71$ & $3.94 \pm 0.87$ & 0.194 \\
\hline \multicolumn{8}{|l|}{ Welfare } \\
\hline Access to psychological support & $3.46 \pm 1.01$ & $3.45 \pm 1.10$ & $3.46 \pm 0.92$ & 0.867 & $3.55 \pm 0.89$ & $3.39 \pm 1.09$ & $0.039^{\dagger}$ \\
\hline \multicolumn{8}{|l|}{ Influence by peers/seniors } \\
\hline SI chosen by peers & $3.48 \pm 1.09$ & $3.54 \pm 1.10$ & $3.45 \pm 1.07$ & 0.249 & $3.77 \pm 0.87$ & $3.32 \pm 1.17$ & $<0.001^{\dagger}$ \\
\hline
\end{tabular}

${ }^{*} \mathrm{p}$-value was calculated using $t$-test. $\mathrm{t} \mathrm{p}<0.05$ was considered statistically significant. SI: sponsoring institution

Other highly rated factors were students' experience during postings, case quantity and variety, work-life balance and working climate. For medical students, clinical rotations are the only time during which realistic impressions of each hospital system are formed. Interactions with faculty and existing residents during these times play an important role in deciding if the institution and the candidate are a mutual fit. Fulfilling requisite training needs by managing a variety of medical conditions is tantamount to training success. Nonetheless, a positive working environment and collegial working relationships among fellow residents and faculty were valued just as much, if not more, by the medical students. A survey on emergency medicine applicants by DeSantis and Marco revealed that friendliness and environment were the two highest-rated factors, ahead of academics. ${ }^{(4)} \mathrm{A}$ study on medical students' choice of an internal medicine programme found house staff morale to be the most important factor. ${ }^{(2)}$ As residency training periods can be very demanding physically and psychologically, a positive working environment with good peer support mitigates burnout. ${ }^{(10)}$

The least important factors in our study had to do with publicity, research opportunities and infrastructure, and quality of welfare and administrative facilities. Methods of reaching out to candidates, namely social media, email or open houses, are generally one-off events with short-term effects. However, these publicity channels may be the students' first encounter with an SI. Rather than selling the programme to potential applicants, it may be more effective to offer quality opportunities to students by directly engaging them in the programmes, as readily available structured internship or elective rotations. Research was also viewed as a factor that was less important, although certain programmes mandate the completion of a research project upon finishing training. We speculate that this may be because local 
hospitals are still predominantly founded on service. Especially for junior staff, research comes at the expense of personal time, as clinical work still takes precedence. This, in addition to the intense rigour of training and tight pace at which residents have to complete postgraduate examinations, may make research a lower priority compared to examinations and clinical work.

There were minimal gender differences among the factors investigated. Female students valued a positive working environment more than male students, although both genders rated these factors very highly. The major differences between the senior and junior students were the perceived importance of research, publicity and work environment. A possible explanation for why juniors valued research more than seniors could be due to the relative balance of clinical and basic science. Junior preclinical students may find research more fresh and interesting, as it ties in with their basic science learning, while clinical students find it challenging enough to cope with learning clinical medicine and preparing for examinations, and thus are less interested in research. On the other hand, having spent time in the wards, it becomes evident to clinical students that working relationships between colleagues is an important factor in the quality of working life.

Our study had a number of limitations. As the study subjects were all medical students and this was a cross-sectional study, we could not ascertain how attitudes changed as these students progressed into residency. The response rate of $55.3 \%$ may mean that selection bias was present; the characteristics of the non-responder group were largely unknown. Additionally, significantly more final-year medical students completed the survey than from any other year. Although this may have skewed the results, it also gives us a better understanding of attitudes among this group of students for whom the change in postgraduate medical education is most relevant, as the decision to enter residency is most imminent for them. As our questionnaire was designed to explore the breadth of factors that may affect the choice of SI, we did not describe in detail terms such as 'mentorship', 'reputation' and 'work-life balance', which are multifaceted, difficult to define and may be broken up into multiple separate factors. We acknowledge that there is significant variability in the interpretation of these terms. Nevertheless, this study has highlighted aspects of residency that are important to students, and we hope that it may serve as the road map for future studies that focus on these specific areas. There was also no data on the participating students' desired choice of specialty, which may have given additional insight into differences in how these factors are valued among students who are inclined towards surgical or non-surgical disciplines.

In conclusion, the vast majority of students felt that quality of education, mentorship, experience as a medical student during rotations and a positive working environment were the most important factors determining their choice of SI. SIs can benefit from taking steps to nurture a culture of mentorship, improve their educational programmes, and foster a healthy and positive work environment to attract future residents.

\section{REFERENCES}

1. Huggan PJ, Samarasekara DD, Archuleta S, et al. The successful, rapid transition to a new model of graduate medical education in Singapore. Acad Med 2012; 87:1268-73.

2. Aagaard EM, Julian K, Dedier J, et al. Factors affecting medical students' selection of an internal medicine residency program. J Natl Med Assoc 2005; 97:1264-70.

3. Mayer KL, Perez RV, Ho HS. Factors affecting choice of surgical residency training program. J Surg Res 2001; 98:71-5.

4. DeSantis M, Marco CA. Emergency medicine residency selection: factors influencing candidate decisions. Acad Emerg Med 2005; 12:559-61.

5. Donovan A, Donovan J. Mentorship in postgraduate training programmes: views of Canadian programme directors. Med Educ 2009; 43:155-8.

6. Hsu AK, Tabaee A, Persky MS. Mentorship in otolaryngology residency: the resident perspective. Laryngoscope 2010; 120:1263-8.

7. Sambunjak D, Straus SE, Marusić A. Mentoring in academic medicine: a systematic review. JAMA 2006; 296:1103-15.

8. Jackson VA, Palepu A, Szalacha L, et al. "Having the right chemistry": a qualitative study of mentoring in academic medicine. Acad Med 2003; 78:328-34.

9. Palepu A, Friedman RH, Barnett RC, et al. Junior faculty members' mentoring relationships and their professional development in U.S. medical schools. Acad Med 1998; 73:318-23.

10. Ishak WW, Lederer S, Mandili C, et al. Burnout during residency training: a literature review. J Grad Med Educ 2009; 1:236-42. 